\title{
Nuevos y enigmáticos grabados rupestres en el sector oriental de la meseta castellano-leonesa (San Esteban de Gormaz, Soria, España).
}

\author{
Juan A. Gómez-Barrera y Elena Heras Fernández
}

Con la publicación de los Grabados rupestres postpaleolíticos del Alto Duero (Museo Numantino, Soria, 1992) dábamos por concluído uno de los capítulos más importante del estudio del arte rupestre del extremo oriental de la meseta castellano-leonesa. El trabajo - Tesis Doctoral de uno de nosotros dirigida por el Prof. Dr. D. E. Ripoll— recogía más de 2.769 figuras grabadas en 73 abrigos rocosos y, a juicio del recensionista de International Newsletter on Rock Art (INORA núm. 5, 1993, p. 31), venía a constituir un trabajo de base para el conocimiento de este tipo de manifestaciones en la Península Ibérica. La investigación, sin embargo, no se estancó y la casualidad ha querido que, pasado un tiempo, fuéramos advertidos de la existencia, en San Esteban de Gormaz (Soria), de una cueva que disponía en sus paredes y techumbre de un amplio, y enigmático, conjunto de grabados rupestres. ¿Enigmáticos? Sí. La cueva «Las Salinas» -como fue bautizada por los niños que la descubrieron- presenta un corpus grabado próximo, por su técnica, estilo y temática, al mundo «esquemático" peninsular si bien la existencia entre sus motivos de, al menos, dos proboscidios provoca en nosotros la duda no sólo ya sobre su adscripción estilístico-cultural sino, y sobre todo, sobre su autenticidad. Precisamente estas dudas son las que -además del consejo de nuestro maestro el Prof. E. Ripoll- motivan las páginas que siguen, en espera de que la experiencia de otros colegas nos pueda dar alguna luz que las disipe o matice.

\section{LA CUEVA}

Se localiza en el paraje de Peña Magdalena, próximo al caserío de la localidad ribereña, en un cortado de la ladera de las estribaciones montañosas 
de aquélla. Oculta por restos de construcciones en piedra y vegetación asilvestrada, se nos muestra en forma de abertura irregular curvada de $3,90 \mathrm{~ms}$. de longitud por no más de $70 \mathrm{cms}$. de altura. Traspasada la entrada, aparece una estrecha $-110 \mathrm{cms}$.de anchura máxima- y prolongada galería cuya escasa altura - nunca superior a la de la boca- la hacen transitable sólo a gatas y por espacio de seis o siete metros. Su forma alargada es irregular en su techumbre y paredes y el suelo actual está conformado por arena fina de sedimentación, tal vez resultante de la acción erosiva del agua de ella manable y estancada, cual pozo, en el espacio exterior inmediato a la oquedad. Para Sancho Marcén y Peña Monne - geólogos de la Universidad de Zaragoza que han realizado el estudio geomorfológico - se trataría de una cavidad kárstica de formación miocénica.

La existencia de la cavidad era conocida desde antiguo por el propietario de los terrenos donde se ubica, mas su reducido tamaño y el pozo - permanentemente cubierto de agua - abierto ante su entrada impidió que accediera a su interior. Las sequías recientes han desecado el lugar y facilitado la entrada, esporádicamente, de un grupo de muchachos que observaron los grabados y se lo hicieron saber - hace ya cuatro o cinco años- a su escéptica maestra. Nosotros conocimos la cueva a través del Dr. Argente Oliver (Director del Museo Numantino de Soria) en octubre de 1993 y desde entonces, y gracias a una pequeña subvención de la Consejería de Cultura y Turismo de la Junta de Castilla y León, venimos estudiándola (Fig. 1).

\section{LOS GRABADOS}

El interés de la cueva radica, obviamente, en los grabados que a ambos lados de la galería (Fig. 2) y en su techumbre pueden observarse. Llaman éstos la atención por su buena conservación sobre todo si se considera que han elegido como soporte paredes que fácilmente se desmoronan con un leve roce. En la zona más interior de la cavidad, esta sensación de fragilidad es menor ya que los grabados se trazan sobre una superficie más dura y presentan una cierta patinación externa que parece concederles más crédito sobre su antigüedad; por contra, los más externos multiplican las dudas respecto a su época de génesis.

En el estado actual de análisis podemos determinar la existencia de, al menos, diecinueve paneles - con una o varias figuras-, grupos, motivos o conjunto de motivos que se ubican, como queda dicho, a izquierda (nueve paneles) y derecha (siete) de la galería y en su techumbre 

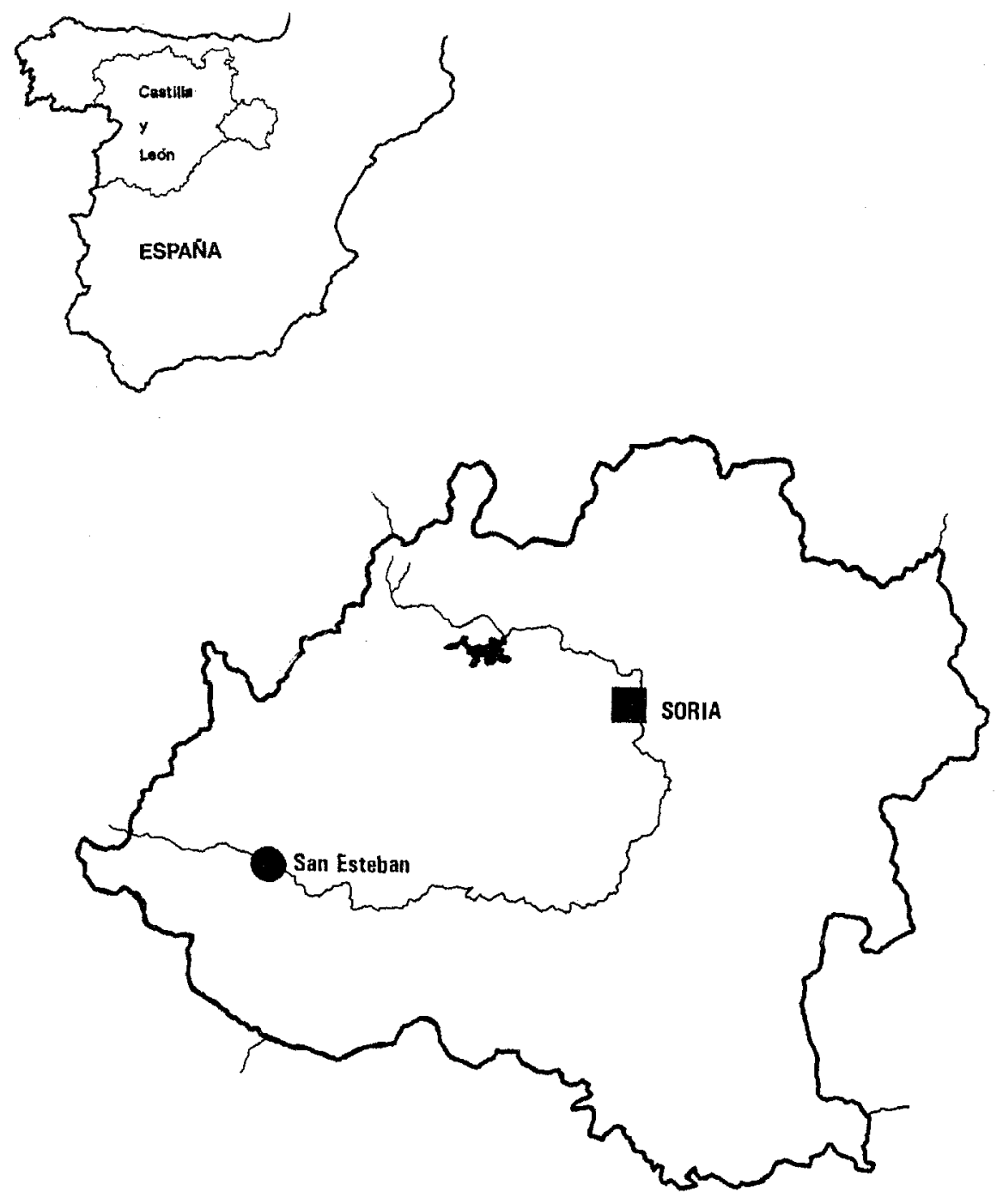

Figura 1. Localización de la cueva "Las Salinas" en San Esteban de Gormaz (Soria, España). 


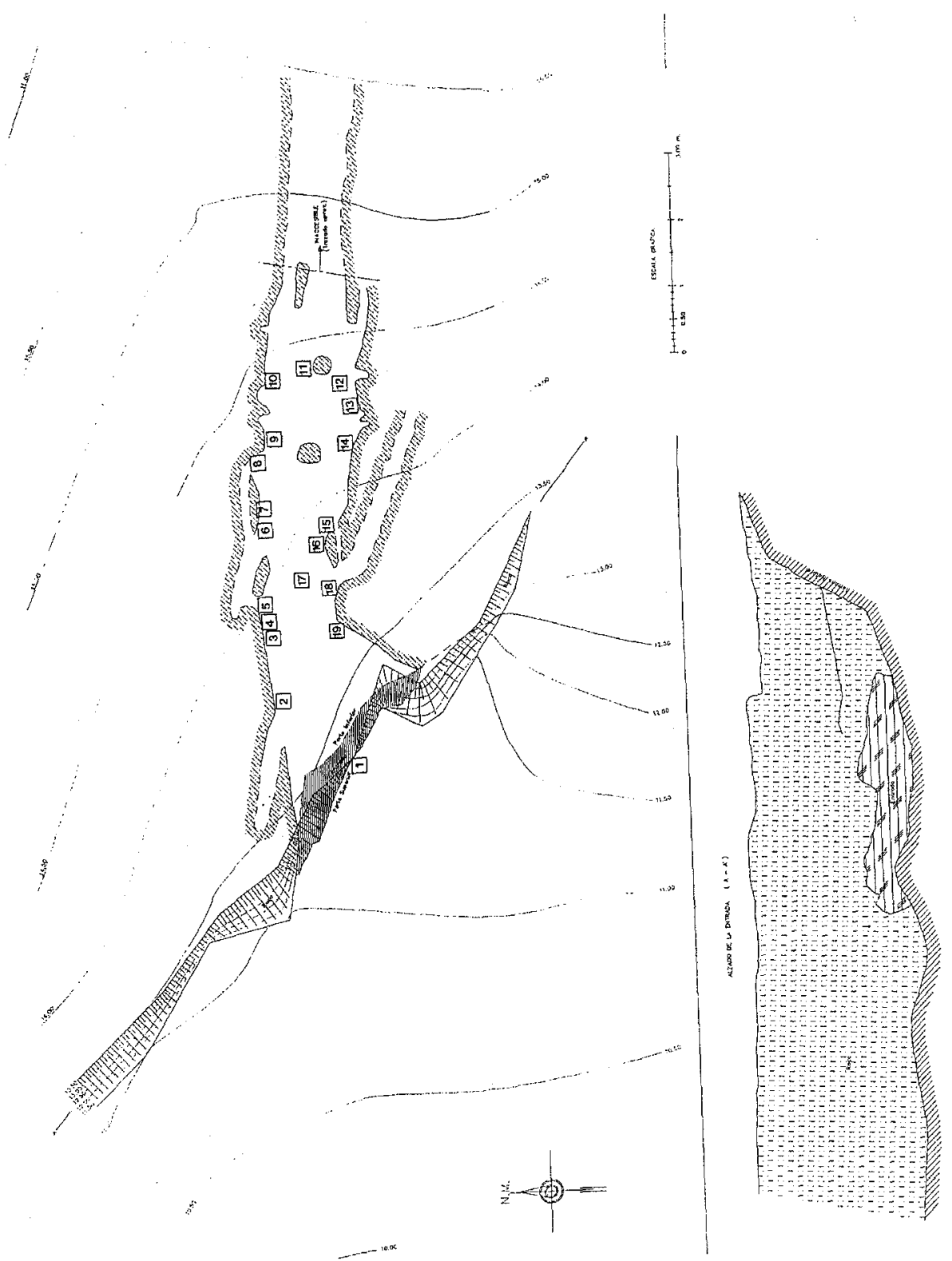

Figura 2. Planta, alzado de la cueva y situación de los grupos grabados. Dibujos de B. Vargas. 
(elefante y arqueros) y entrada de la cueva. Diecinueve paneles que parecen repetir, entre figuraciones de abstracción lineal y geométrica, toda una serie de antropomorfos, de silueta más o menos aspada, algunos provistos de arco, y cierto dinamismo en su trazado (Fig. 3).

Junto a estas representaciones de carácter esquemático sorprende la presencia de varios cuadrúpedos de los que, al menos dos, nos recuerdan a proboscidios o, más exactamente, a sendos ejemplares de la familia de los elefántidos. Se dibujan en grabado fino, marcando con rotundidad sus trompas y defensas. sus anchos cuerpos, sus patas y sus ojos, uno por ejemplar. El primero de ellos, el más cercano a la entrada (Fig. 4), aparece acompañado de otros motivos mientras que el segundo se localiza en el interior de la cavidad, en el techo, a tan sólo $40 \mathrm{cms}$. del suelo actual - cosa ésta que además de dificultar su contemplación debió complicar mucho su ejecución - y totalmente aislado (Fig. 5). Mide $13 \mathrm{cms}$. y a simple vista ofrece diferencias estilísticas (diseño del perfil dorso-lumbar, fundamentalmente) con el primero. ¿Son contemporáneos? ¿Se realizó el primer ejemplar a la vez que el resto de los grabados tras observar el

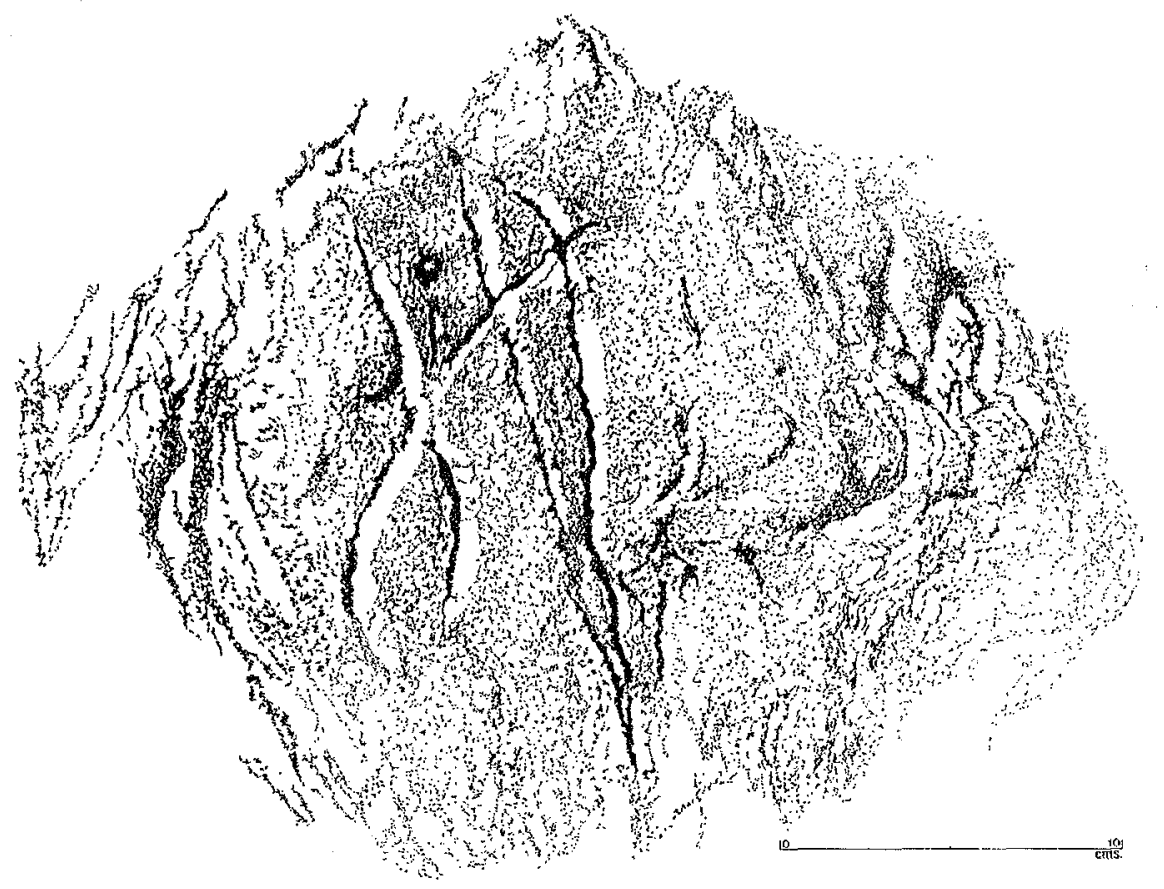

Figura 3. Arquero del Grupo 10. 


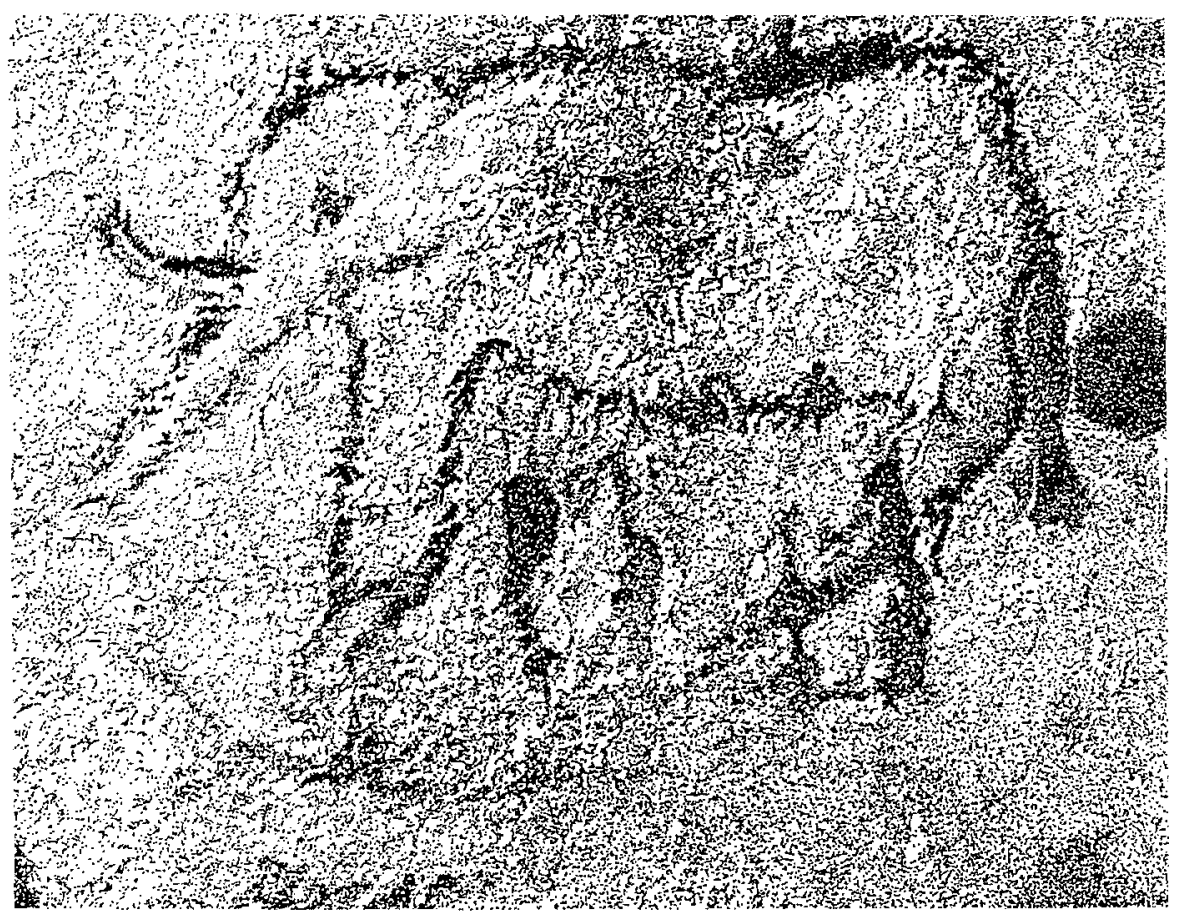

Figura 4. Mamuth o elefante del conjunto grabado núm. 11.

elefante/mamuth aislado del interior? ¿Se grabó este último después? ¿Estamos ante dos autores distintos? Mucho nos tememos que sean éstas cuestiones sin respuesta posible.

Salvo un inicial tizonazo en forma de "T», de aspecto y apariencia reciente pero susceptible de ser analizado por C-14, el conjunto plástico de «Las Salinas» ha sido realizado en técnica de grabado.

La superficie rocosa - arenisca muy alterada- determina un soporte artístico blando, fácil de ser grabado por leve incisión o reiterada frotación.

Con matizaciones más especíícas que puedan posteriormente hacerse, hemos observado hasta ahora dos tipos de trazos grabados, a saber: uno ancho (de 1 ó $1,2 \mathrm{cms}$.), resultado, tal vez, de una primera ejecución en incisión en «U» y posterior reiteración abrasiva; otro más fino (de 0,2 a $0,4 \mathrm{cms}$.) trazado, seguramente, sólo en incisión en «U», con un útil 
no demasiado duro — basta la presión de la uña para dejar huella similar a los grabados referidos- y posiblemente no metálico, aunque no tenemos ningún juicio de valor suficientemente contrastado para confirmar tal aserto. El primer trazo grabado podría incluir los grabados del lateral izquierdo de la galería así como los motivos del conjunto núm. 13 situado a la derecha, especialmente los arqueros en aspa. El grabado fino ha sido utilizado en los proboscidios y en los arqueros del techo, si bien la mayor dureza de las areniscas del nivel superior que alberga a éstos pudo ser causa del menor tamaño en el grosor de la incisión.

El tamaño de las figuras oscila entre los $20-30 \mathrm{cms}$. de alguno de los arqueros aspados -incluyendo la prolongación del arco-y aquellos otros del techo que no superan los $7 \mathrm{cms}$. Los cuadrúpedos, por su parte, se sitúan entre los 10-13 cms. aproximadamente.

\section{EL PROBLEMA}

Las técnicas del grabado observadas en «Las Salinas» son, en líneas generales, las admitidas como comunes en los grabados postpaleolíticos en cuevas de la provincia artística Centro-Norte de la Península Ibérica (Gómez-Barrera, 1992a). Incisión y abrasión son utilizadas, con cierta exclusividad, en Cueva Maja (Cabrejas del Pinar), Covarrubias (Ciria) y cuevas de San Bartolomé (Ucero) por citar tan sólo los ejemplos sorianos (Gómez-Barrera, 1992b). El repiqueteado, tan frecuente en los grabados al aire libre, no queda reflejado, a nuestro modo de ver y entender, en ninguno de los motivos de la cueva.

Es, pues, a este momento (Calcolítico-Bronce Antiguo) del grabado rupestre postpaleolítico al que nos queremos inclinar al menos para encuadrar los motivos abstractos y figurados no animalísticos. El problema de los proboscidios, e incluso de otros zoomorfos no bien identificados, nos supera en estas cuestiones.

El arte rupestre prehistórico peninsular —obviamos así el arte mueble de Las Caldas: Corchón, 1991-1992- tan sólo conoce tres ejemplares de proboscidios: los del Pindal (Pimiango, Asturias), El Castillo (Puente Viesgo, Cantabria) y Ojo Guareña (Sotoscueva, Burgos). Y los tres parecen pertenecer a la familia de los mamútidos. El Prof. Ripoll añade un cuarto mamuth representado en la cueva de Los Casares, en Riba de Saelices (Guadalajara), a la vez que descarta que los motivos del Pindal y del Castillo pertenecieran al tipo de elefante de colmillos rectos (Palaeoloxodon antiquus) que habría persistido en la Península Ibérica hasta el Paleolítico 
Superior. El Mammuthus primigenius, según el propio investigador (Ripoll Perelló, 1989), viviría en Europa durante toda la glaciación de Würn y al final de los tiempos glaciares emigraría hacia las regiones septentrionales frías del continente euroasiático.

Leroi-Gourhan informa (1983) que el mamuth, que tan sólo alcanza un $9,3 \%$ del total de los animales grabados, pintados y esculpidos del arte paleolítico, está representado de manera desigual pero constante en todas las regiones, salvo en el Centro y Sur de España e Italia. $Y$ sin embargo supera el centenar de unidades en Rouffignac (Perigord) y es el animal predominante en los yacimientos franceses de Arcy-Sur-Cure, Bemifal, Pech-Merle y Chabot.

Se hace difícil entender, por tanto, la presencia de proboscidios (y en tan alto número) en la cueva soriana de "Las Salinas", si no nos remontamos al Paleolítico Superior... o si no concluímos que estos motivos, de trazado en principio diferente del resto de las figuras grabadas de la cueva, son falsos o, dicho de otro modo, de ejecución moderna.

¿Y los arqueros? No son muy frecuentes entre los grabados rupestres postpaleolíticos. Son muy abundantes, casi diríamos significativos, en la pintura rupestre levantina. $Y$ aunque ni en Valonsadero ni en Oteruelos, Pedrajas, Fuentetoba, Ligos y Ucero aparecen (Gómez-Barrera, 1982 y 1992c), sí tienen una participación activa en la pintura rupestre esquemática (Acosta, 1968: figs. 48 y 49)

Resulta complicado paralelizar los arqueros de San Esteban de Gormaz (Soria) con los esquemáticos recogidos por P. Acosta. Por su dinamismo y, sobre todo, por la composición escénica de uno de los conjuntos, nos recuerdan más las escenas levantinas de Cueva Remigia (Ares del Maestre), El Roure (Morella la Vieja) o Cueva del Civil (Valltorta). Sin embargo, estamos en presencia en ellos de un arte pictórico, por ahora adscrito a una región concreta (el Levante español) y cuyas figuraciones utilizan soportes al aire libre. Por si fuera poco parece que el grabado aquí tan sólo se utiliza para formatear la línea del perfil de los animales que luego serán rellenados con pintura, siendo muy raro - hay quien lo descarta totalmente del ciclo artístico levantino- su aparición aislada, aunque recientemente A. Sebastián (1988) ha dado a conocer grabados de estilo levantino en Teruel.

¿Estamos ante un yacimiento prehistórico o es una falsificación histórico contemporánea? Si el yacimiento es Paleolítico, como en buena lógica demandarían los proboscidios, ¿qué pintan, en un mismo contexto artístico, los grabados postpaleolíticos? Y si, por contra, lo que observamos es una facies esquemática ¿qué motiva la presencia de aquéllos? ¿Se trataría de 
una reutilización holocénica de un santuario pleistoceno como ha señalado en otras zonas Apellániz (1982)? Incluso, ¿cómo aparece una escena estilísticamente levantina en un lugar tan alejado de la zona que le es propia? ¿Acaso se podría hablar de una facies artística localista paralela al mundo levantino?

Con todo, la cueva «Las Salinas» es, para nosotros, un yacimiento de primer orden en tanto no se dé respuesta a alguna de las cuestiones aquí planteadas, cosa, por otra parte, ciertamente difícil y por lo que esperamos que, cuantos nos lean, nos hagan llegar sus opiniones al Departamento de Arqueología del Servicio Territorial de Cultura de la Junta de Castilla y León en Soria.

\section{BIBLIOGRAFÍA}

Acosta, P. (1968): La pintura rupestre esquemática en España, Salamanca.

ApEllaniz, J. M. (1982): El arte prehistórico del País Vasco y sus vecinos, Desclée de Bouwer, Bilbao.

CORCHON, S. (1991-1992): Representaciones de fauna fria en el arte mueble de la Cueva de Caidas (Asturias, España). Significación e implicaciones en el arte parietal, Zephyrvs, XLIV$X L V$, p. 35-64.

GÓmEz-BARRERA, J.A. (1982): La pintura rupestre esquemática en la Altimeseta Soriana, Soria.

Gómez-BARRERA, J.A. (1992a): Manifestaciones de la facies esquemática en el centro y norte de la Península Ibérica, Espacio, Tiempo y Forma, Serie 1, Prehist. y Arqueol., t. V, p. 231-264.

Gómez-BARRERA, J.A. (1992b): Grabados rupestres pospaleolíticos del Alto Duero, Serie de Investigación, 1, Museo Numantino, Soria.

Gómez-BARRERA, J.A. (1992c): Arte rupestre en la provincia de Soria, en Actas del 2. Symposium de Arqueologia Soriana, Soria, t. 1, p. 9-64.

LEROI-GouRHAN, A. (1983): Los primeros artistas de Europa, Madrid.

RIPOLL. PERELLo, E. (1989): El arte de los cazadores paleolíticos, Madrid.

SEBASTIÁN, A. (1988): Nuevos datos sobre la cuenca media del río Guadalope: el abrigo del Barranco Hondo y el abrigo del Angel, Teruel, 79 (II), p. 77-92. 